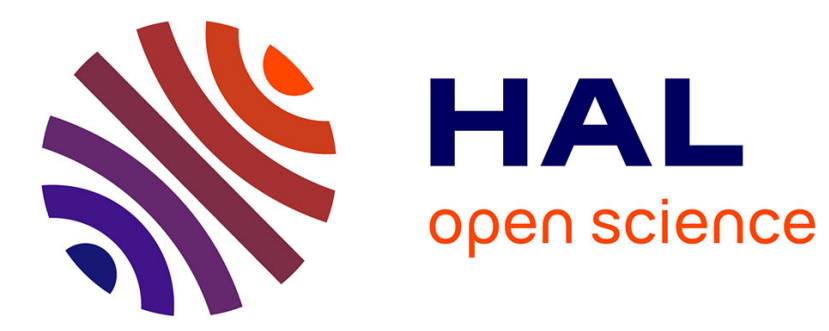

\title{
Sur le rayonnement du radium à la température de l'hydrogène liquide
}

\author{
Pierre Curie, M. Kamerlingh Onnes
}

\section{To cite this version:}

Pierre Curie, M. Kamerlingh Onnes. Sur le rayonnement du radium à la température de l'hydrogène liquide. Radium (Paris), 1913, 10 (6), pp.181-186. 10.1051/radium:01913001006018100 . jpa00242609

\section{HAL Id: jpa-00242609 https://hal.science/jpa-00242609}

Submitted on 1 Jan 1913

HAL is a multi-disciplinary open access archive for the deposit and dissemination of scientific research documents, whether they are published or not. The documents may come from teaching and research institutions in France or abroad, or from public or private research centers.
L'archive ouverte pluridisciplinaire HAL, est destinée au dépôt et à la diffusion de documents scientifiques de niveau recherche, publiés ou non, émanant des établissements d'enseignement et de recherche français ou étrangers, des laboratoires publics ou privés. 


\title{
MÉMOIRES ORIGINAUX
}

\section{Sur le rayonnement du radium \\ à la température de l'hydrogène liquide}

\author{
Par Mme P. CURIE et M. KAMERLINGH ONNES
}

[Laboratoire cryogénique de Leyde.]

Parmi les proprictés des corps radioactifs, l'une des plus remarquables est l'indépendance du rajonnement de la température. On ne constate non plus aucune influence de la tempéralure sur la valeur des constantes radioactives. Ces deux faits sont connexes; ils prouvent que les transformations radioactives ne sont pas sensibles à l'action de la température qui joue un ròle si important dans les Iransfurmations chimiques moléculaires.

Dans la théorie des transformations rudioactives, l'intensité du rayonnement d'une substance simple est, en effit, proportionnelle à la vitesse de transformation, de sorle que la varialion de l'une de ces quantités entraine lx variation de l'autre. Les recherches expérimentales, relatives à l'action de la température, ont porté sur la mesure de constantes radioaclives et sur celle de lintensité du rajonnement de quelques substances. P. Curie a montré que la loi de transformation de l'émanation n'est pas modifiée à la température dec $450^{\circ}$ ou à la température de l'air liquide ${ }^{1}$.

Divers ubservateurs ont constaté que le rajonnement pévétrant du radium ou de l'uranium est le mème à la température ordinaire et à la température de l'air liquide?

L'action des températures élevées sur l'émanation du radium et sur les produils de sa transformation, en particulier sur le radium C, a aussi fiat l'objet de plusieurs travaux a $t$ a donré lieu ì des discussions. Il semble néanmoins lígitime de conclure que les variations obscrvées dans certains cas sont attribuables à des phénomènes accessoires d'importance secondaire et que les constantes radioac!ives des substances indiquées ne sont pas modifiées diune manière appréciable quand la température s'élève jusqu’à 1500 degrés ${ }^{5}$.

Elant donnée la grande importance de celte question, on pouvait chercher à étendre ces résultats en opérant dans les limites de température plus íten-

1. P. Cicrie. C. R. (190j).

2. Bilectrich. (illie. Dewar. Retherford.

5. Cirie et D+w . C. R. (191't : Bunsox. Plell. Mag. (1906).

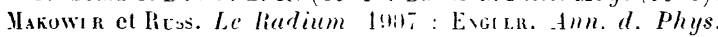
(1908): Schuid, Phys. Zeilschr, 1908,.

T. 10. dues et en augmentant la précision des mesures, laquelle pouvait au plus atteindre 1 pour 100 dans les recherches dont il vient d'ètre question.

Nous nous sommes proposé d'utiliser la température de l'hydrogène liquide. L'emploi d'une méthode de compensation permettait d'apprécier une très petite variation dans l'intensité du rayonnement.

Les mesures ont porté sur le rajonnement pénétrant du radium. Les résultats ne sont pas farorables à l'existence d'un effet rapide de ce grand abaissement de température sur le rajonnement, à une prćcision qui atleint probablement 0,2 pour mille.

Ce travail a été exécuté pendant la première partie de l'année 1911. Les mesures préparatoiras ont été effectuécs d'une part à Paris, d'autre part ì I.eyde. Les mesures définitices ont été faites à Leyde en juillet 1911. Les expériences deraient ètre continućes et étcndues et pour celte raison la publication a été retardée. Mais la reprise du travail ayant été empèchée jusqu’à présent par suite d'une longue maladie de l'un de nous. il semble préférable de publier les résultats obtenus.

Après quelques tàtonnements, le dispositif expérimental suivant a élé adoplé.

L'appareil (fig. 1 et 2 ) se compose d'un vase Dewar $\Lambda$ dans lequel on tait pénétrer un vase de cuivre B contenant le bain froid. Le vase bewar, assez lırge dans sa partie supérieure $A_{1}$, est constitué dans sa partie inférieure $A_{2}$ par un tule dont la longueur est environ $16 \mathrm{~cm}$ et les deur diamètres $8,5 \mathrm{~mm}$ et $15 \mathrm{~mm}$. Le vase de cuive s'applipue bien sur les parois du vase bewar et possède comme celuici une partic tubulaire, moins longue que celle du vase Dewar. Le tube de cuivre est fermé à son extrémité inférieure $B_{1}$ par une pièce métallique $C_{1}$ qui porte un tube d'aluminium mince $\mathrm{C}_{2} \quad(0,5 \mathrm{~mm}$ d'épaisseur!, dans lequel se troure une ampoule de verre contenant le radium. L'espace étroit dans lequel est placée cette anpoult, dans la partie inférieure du vase Dewar, constitue une chambre froide dunt la température diffère très peu de celle du bain froid; la différence pouvait à peine ètre appréciée quand le 
vase de cuivre était rempli d'air liquide. Il a donc paru préférable d'opérer ainsi, plutòt que de placer l'ampoule elle-mìme dans l'air liquide, ce qui présente toujours un certain danger.

Les rayons émis par l'ampoule sont en partie des rayons pénétrants qui peuvent traverser les parois du

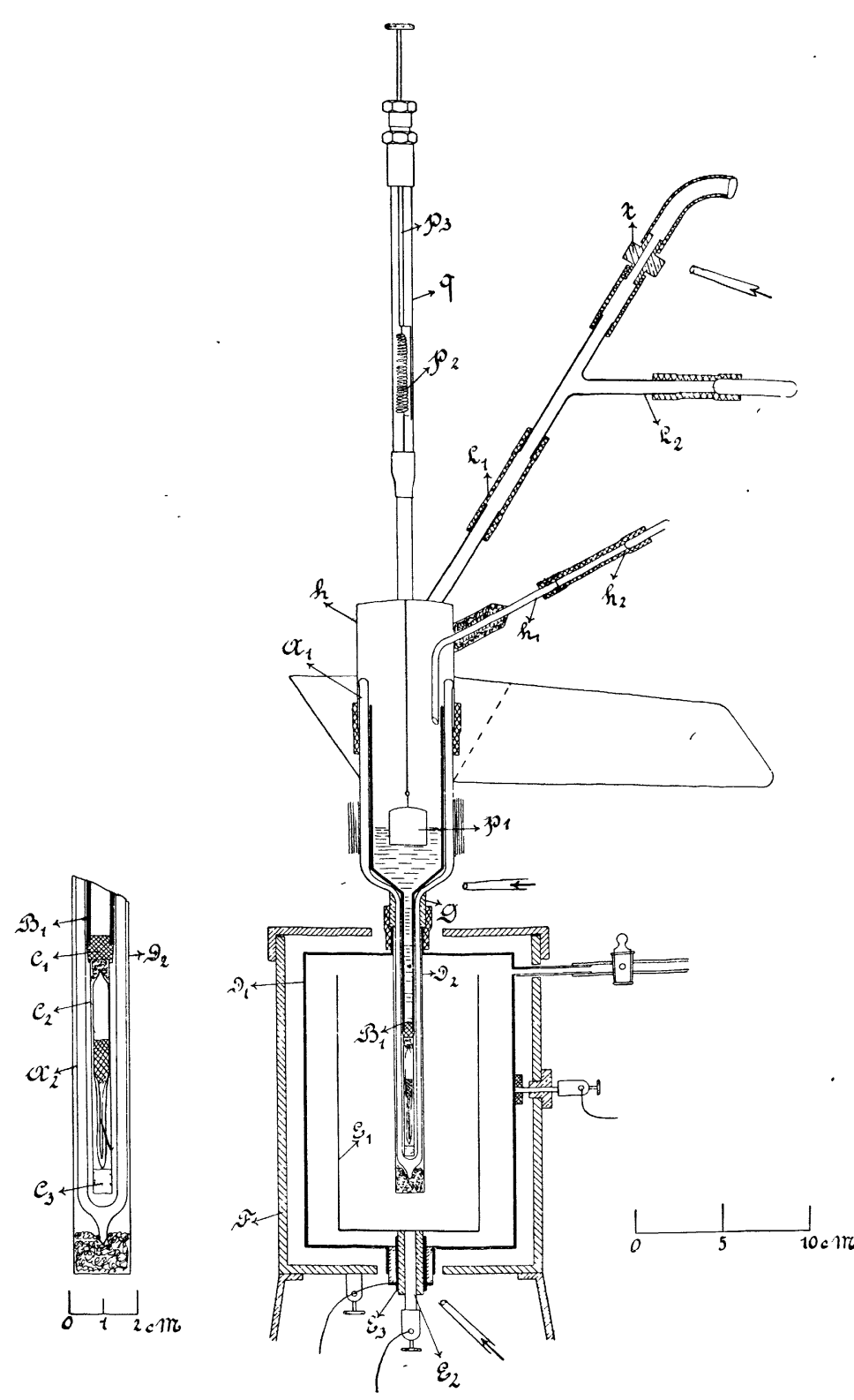

Fig. 1.

tube d'aluminium et celles du vase Dewar et pénétrer au travers d'une paroi métallique dans une chambre d'ionisation étanche. Celle-ci se compose d'une boìte cylindrique $\mathrm{D}_{1}$ relice à une batterie; dans la base supérieure de cette boìte est soudé un tube disposé suivant l'axe du cylindre et fermé à l'extrémilé inlérieure. L'électrode isoléc $E_{1}$, ajant la forme d'un cylindre à fond, est reliée à l'électromètre. La boite métallique $\mathrm{F}$, reliée au sol, assure la protection électrostatique. Quand l'appareil est monté, la partie tubulaire du rase Jewar pénètre dans le tube central $\mathrm{D}_{2}$ de la boîte $\mathrm{D}_{1}$, le raccord étant obtenu au mojen d'un tule de caoutchouc épais s'appliquant sur une pièce d'ambre $g$ mastiquée sur le vase Dewar. L'ampoule étant en place, les ions sont produits dans l'air qui remplit la boìte $\mathbf{D}_{1}$, de part et d'autre de l'électrode E. Le courant recueilli par celle-ci est mesuré au moyen d'un électromètre et d'un quartz piézo-électrique.

L'expérience consiste à mesurer le courant d'ionisation produit par les rajons du radium $1^{\circ}$ quand le radium est à la température ambiante; $2^{\circ}$ quand le radium est à la tempéralure de l'hydrogène liquide.

La chambre d'ionisation qui est extéricure au vase Dewar reste d'ailleurs sens:blement à la température ambiante. Cette chambre est étanche, et la masse de gaz qui y est contenue ne varie pas pendant les expéricnces.

On augmente grandement la sensibilité de la méthode si, au lieu de mesurer la totalité du courant, on utilise une méthode de compensation. Celle-ci consiste à compenser le courant à mesurer par un courant de sens opposé, produit dans une seconde chambre d'ionisation par les rayons pénétrants d'une ampoule de radium qui ne subit aucune variation de température pendant les expériences. Ce compeusateur de courant est d'un type ordinaire dans les mesures de radioactivité. L'électrode isolée G. a la forme d'un tube fermé à l'extrémité inféricure et rulié à l'électromètre et à l'électrode $\mathrm{E}_{1}$ au mojen de fils protégés par des tubes de láiton remplis de paraffine. Le tube G pénètre à l'intéricur d'une boìte cylindrique $\mathrm{H}$ reliée à une batterie et formant chambre d'ionisation. La boite extérieure $\mathbf{K}$ sert d'enveloppe protectrice. L'électrode $\mathrm{G}$ (de même que l'électrode $\mathrm{E}_{1}$ ) est protégće à la manière ordinaire par un tube de garde relié au sol. A l'intérieur du tube G se trouve une ampoule qui contient un sel de radium. Les boîtes $\mathrm{D}_{1}$ et $\boldsymbol{H}$ sont maintenues à des potentiels élevés de signes contraires. Ilans ces conditions, on mesure à l'électromètre la différence des courants d'ionisation produits dans les deux chambres. Avec des courants 
d'intensité suffisante on peut ainsi atteindre une sensiỏilité très élevée.

Il est utile de remarquer que diverses petites imperfections des méthodes de mesures, qui restent d'ordinaire inaperçues, deviennent visibles quand on opère de la manière qui vient d’ètre décrite. Ainsi, du sel à l'aide de secousses répétées. Mais on laisse néanmoins subsister de petits effets de ce genre inférieurs à 1 pour 1000 dans nos expériences. La plus grande prudence est donc nécessaire dans les manipulations nécessitées par les expériences.

Les oscillations radioactives du courant d'ionisation

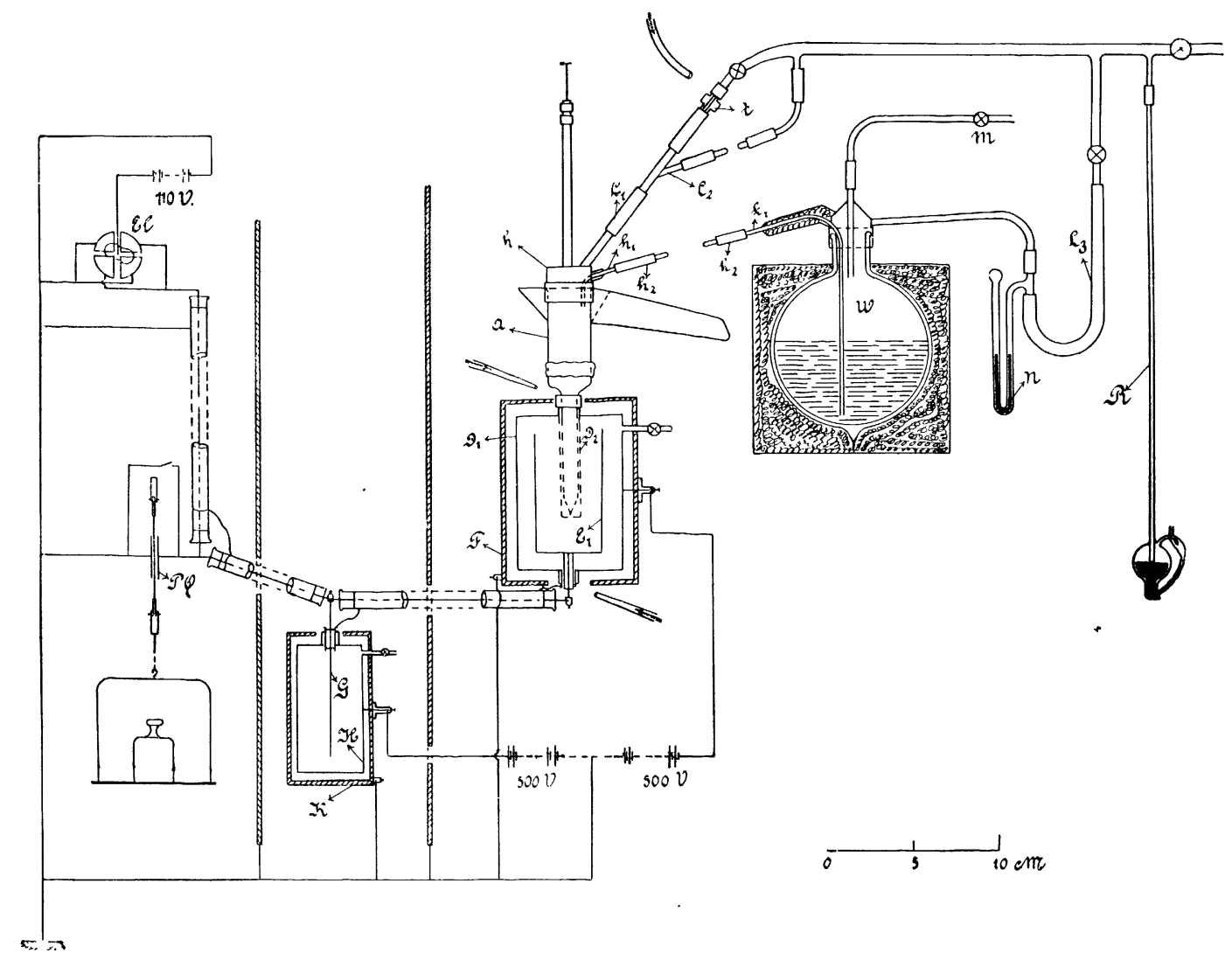

Fig. 2.

quand on mesure chaque courant séparément, la saturation semble complète pour des potentiels de כ00 volts. Mais, en mesurant la différence des courants, laquelle n'alteignait généralement pas 5 pour 100 de la va'eur de chacun d'cux, on pourait se rendre compte que le courant dans chaque chambre augmentait encore avec le voltage. Quand

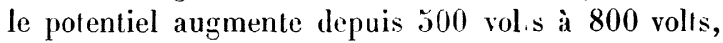
le courant augrmente de 2 à $\mathbf{5}$ millièmes. Il faut donc opérer avec des voltages invariables.

La précision est limitée par les conditiuns de stabilité des apparcils et par les oscillations radioactives.

Le travail a été effectué ares des sels de radium solides, contenus dans les ampoules. Le sel était en petits grains, et l'ampoule n'était pas complèiement remplie. Quand des secousses se produisent, les grains peuvent se déplacer dans une certaine mesure. Il en résulte un petit changement dans la distribution du råonnement à l̈intéritur des chambres. On en diminue le dange $r$ en réalisant d'arance un tassement se manifestent quand on augmente sulfisamment lit sensibilité des mesures. Elles donnent lieu à des irrégularités qui ne peuvent ètre éliminées que par un grand nombre de mesures. Elles sont le moins à criandre quand on opère avec les rayons $\gamma$, ce qui était le cas dans nos expériences. Elles ne pouvaient apporter de troubles dans les mesures.

Il est très important que les chambres d'ionisation conliennent une masse d'air invariable. Quand on utilise des rajons pénétrants, le courant est, au moins approximativcment, proportionnel à la masse d'air ionisé. Pour que le courant reste constant, à une grande précision, il faut donc assurer la fermeture parfaite des chambres. Chacune d'elles était munie d'un robinet. En faisant varier la masse d'air dans la chambre de compensation, on pouvait régler le courant dins cette chambre, de manière à obtenir la compensalion au degré voulu.

L'air sec est introduit dans les deux chambres au moyen d'un tube rempli d'ouate, qu'on relie par 
un joint rodé au robinet de la chambre, à une pompe à vide et à un manomètre qui permet de régler l'admission.

Tous avons dù prendre beaucoup de soins pour éviter que les opérations crjogéniques n’entrainent des défauts d'isolement, par suite d'un dépôt d'humidité de l'atmosphère sur les parties refroidies de l'appareil. L'arrangement cryogénique adopté a permis d'éviter toute difficulté de ce genre. Cet arrangement qui, du rcste, était indiqué pour travailler facilement et en toute sécurité avec l'hydrogène liquide, avait aussi cet arautage que l'ampoule à radium ne pouvait être en contact quavec le gaz du bain, de sorte qu'on n'avait pas à craindre des dépôts d'air solide autour d'cl'e.

L'appareil cryogénique est tout à fait fermé. Le vase Dewar porte un couvercle $h$, d'argentan mince, qui y est appliqué avec une ligature en caoutchouc. permettant de réaliser une fermeture hermétique, de sorle que, l'ampoule avec lı radium étant mise en place, tout l'appareil cryogénique peut être évacué et rempli d'avance (par $l_{1}$ ) avec de l'hydrogène gazeux pur el sec.

Une perforation $\mathrm{du}$ petit bouchon $\mathrm{C}_{5}$, sur lequel l'ampoule repose dans le tube d'aluminium, assure l'équilibre de pression, qui s'y établit facilement pendant cette opération, de sorte que l'ampoule ne court aucun risque par cet effet.

L'hjdrogìne liquide (st introduit dans le vase B, par le petit tube en argentan $h_{1}$, et le tube cn caoutchouc $h_{2}$. Pour cela, on cnlève le bouchon en verre qui ferme celui-ci, et on $\mathrm{y}$ inlroduit (après avoir enlevé le bouchon $k_{2}$ ) le tube siphon $k_{1}$ d'une grande boule Dewar $w$, dans lequel on a transporté l'bydrogène liquide préparé d'avance. Avant de faire le raccord du siphon et du tube, l'appareil et la boule sont mis en communicalion par les tubes $L_{1}$ et $L_{5}$ arec un gazomètre d'hydrogène pur. Le raccord élant fait, on supprime la communication de la boule et du gazomètre et, en exerçant une pression sur le robinet $m$, à l'aide d'un réserroir à hỵdrogène comprimé, d'après les indications d'un manomètre ì mercure $n$, on verse l'lyydrogène liquide dans l'appareil. La communication entre la boule de provision et le gazomètre est alors rétablic, le raccord réchauffé, le siplion séparé du tube, et cclui-ci de nouveau fermé par le bouchon de verre aussitòt remis en place. Pour éviter que ces manipulations ne donnent des sccousses it l'appareil, le tube en caoutchouc $h_{2}$, d'ordinaire aussi court que possible, a été pris assez long; mais comme le caoutchouc devient très cassant par le froid extrême, ce qui peut occasionner des ennuis, nous nous sommes bornés à une longucur de $7 \mathrm{~cm}$. Les secousses sont restées alors au-dessous des limites de stabilité de l'appareil qui a servi aux expériences, dont il est question dans ce mémoire.
Dans un plus grand appareil, destiné à des expériences prolongées pendant plus de vingl-quatre heures, avec lequel nous n'avons encore pu faire que des expériences préliminaires, nous avons réussi à obtenir une plus grande stabilité, et nous sommes devenus plus indépendants des secousses causées par les manipulations. En versant le gaz liquéfié, il faut éviter qu'il ne déborde du vase en cuivre $B$, auquel cas il pourrait pénétre $r$ dans la chambre fruide, ce qui donnerait lieu à des irrégularités et pourrait nuire à l'ampoule. D'un autre côté, il faut être averti quand le bain est évaporé, afin de ne pas prolonger les expériences sans ètre sùr de la température. La hauteur du nircau du gaz liquéfié cst appréciée au moyen d'un flo:tcur. C'est une boîte d'argentan $p_{1}$ suspendue à un faible ressort $p_{2}$ porté par une tige $p_{3}$. La tige glisse dans une boite d'étoupe qui se trouve à l'extrémité supérieure d'un tube en verre $q$, porté par le couvercle $h$. A côté du ressort ct suspendue avec lui à la tige, se trouve une pelite échelle, munie en bas de quelques divisions. Vu la densité très petite $(1 / 14)$ de l'hydrogène liquide, le flolteur est très léger.

Lorsqu'en baissant le ressort, le flotteur attcint la surface, on s'en aperçoit par un raccourcissement du ressort et on lit la hauteur du nireau sur la division et sur la tige.

Lorsqu'on veut verser le gaz liquéfié, on règle li position du flottcur au niveau choisi pour le rempli:sage.

Avant de commencer les nesures on infunce le ressort de manière à être averti par so.l allongement quand le bain est éraporé jusqu'au niveau auquel on veut arrêter les expériences.

L'hydrogène évaporé s'échappe par $\mathrm{L}_{1}$. Le tube $\mathrm{R}$, se terminant sous du mercure, fonclionre comme tube de sûreté.

Pour assurer l'isolement du vase Dewar et pour éviter des courants nuisibles à la constance de la batterie, le tube $\mathrm{L}_{1}$ est interrompu par une pièce d'ambre t. On évite que celle-ci se refroidisse trop pendant le remplissage en dirigeant alors les vapeurs froides par un conduit auxiliaire $\mathrm{I}_{2}$ qu'on supprime après que le gaz liquéfié a cité versé. Le débit de vapeur étant derenı normal, un courant d'air un peu plus chaud que celui de la salle suffit pour maintenir l'isolement. l.e courant d'air est fourni par un réservoir à air comprimé; l'air passe dans un long tube dont une partic est chauffée par de l'eau chaule.

Le raccord de la pièce d'ambre $g$ mastiquée sur le vase Dewar arec le lube $D_{2}$ de la chambre principale est fait arec beaucoup de soin, pour assurer une fermeture hermétique, de sorte que l'humidité ne puisse pénétrer dans l'espace libre entre le tube et le vase.

Au moyen d'une gaine de papier, on dérie les cou- 
rants d'air froid qui descendent. Il faut aussi tenir compte de l'eau qui en venant du courercle coule le long du verre. Le froid intense des vapeurs à l'intérieur du couvercle détermine sur celui-ci, pendant le remplissage, un dépôt de givre qui fond ensuite. Ie plus, quand le remplissage est terminé, la condensation d'humidité atmosphérique continue en donnant de l'eau; celle-ci est prise par de la ouate au-dessus de la gaine de papier dont nous venons de parler et au-dessous de celle-ci par du papier buvard. Sur la pièce d'ambre on dirige un courant d'air sec un peu chauffé, qui en même temps sèche le bas du vase Dewar.

Il reste à empècher le refroidissement des pièces par lesquelles Y'électrode princip ıle $E_{1}$ communique avec l'électromètre. Pour cela on dirige de mème un courant d'air sec et légèrement chauffé sur le bouchon d'ambre entre la tige $\mathrm{E}_{2}$ et l'anneau de garde $\mathrm{E}_{3}$ en bas de la chambre principale.

Les courants d'air refroidi qui descendent des conduites de gaz s'snt déviés loin des appareils par des écrans et des gaines appropriées. Les mouvements d'air dans la salle ont été cmpêchés autant que possible afin d'éviter un déplacement d'air ionisé autour des contacts, protégés du reste par plusieurs écrans en plomb, indiqués schématiquement, par des l'euilles d'étain, etc. L’influence des courants de chauffage a éié essayée à la température ordinaire et ne donnait lieu à aucun efiet d'influence.

Expériences préliminaires. - La méthode expérimentale a d'abord été éludiée à Paris, en ulilisant de l'air liquide pour le bain froid.

Le courant dans la chambre principa'c était obtenu au moyen d'une ampoule contenant environ $0.1 \mathrm{gr}$. de chlorure de radium. Dans la chambre compensatrice l'on utilisait une ampoule contenant environ 23 mgr. de chlorure de radium.

Dans les premières expériences, l'ampoule principale itait contenue dans un tube d'aluminium de $0,3 \mathrm{~mm}$ d'épaisseur, et le tube central $\mathrm{I}_{2}$ de la chambre $\mathrm{D}_{1}$ élait également en aluminium, de $0,5 \mathrm{~mm}$ d'épaisscur. Les rajons traversaient, avant de pénètrer dans la chambre, une épaisscur d'aluminium de $0,8 \mathrm{~mm}$ en tout, ainsi qu'une épaisseur de verre d'environ 2, à mm (paroi de l'ampoule et les deux parois du vase Dewar).

Lors du rufroidissement, on observait toujours une diminution du courant dans la chambre principa'e. Cette diminution, assez peu régulière, était d'environ 2 pour 100 , elle s'établissait rapidement, dès que l'air liquide était versé dans le vase de cuivre, et atteignait sa valeur maximum en une demi-hrure environ.

Cependant, en retirant rapidement l'air liquide du vase et en suivant, au moven d'un couple thermo- électrique, la température de l'ampoule, on pouvait constater que cette température restant encore fixe, le courant remontait déjà, pour reprendre sensiblement la valeur primitive après le réchauffement complet de l'appareil. On pouvait conclure de là que la diminution de courant observée n'était pas attribuable à une variation du rayonnement de l'ampoule, mais à quelque autre circonstance.

Après divers essais, il a paru probable qu'il s'agissait d'un changement de l'effet absorbant des écrans par suile de leur contraction à basse température. Il a donc paru nécessaire d'utiliser des éc:ans plus denses et plus épais, afin d'opérer avec les rayons les plus pénétrants seulement, sur lesquels des effets de ce genre sont moins à craindre. En entourant l'ampoule d'un tube de cuivre de $1 \mathrm{~mm}$ d'épaisseur, on a constaté, en effet, que la diminution de courant à l'introduction de l'air liquide se trouvait réduite à 0,1 pour 100 seulement; elle était établic en 10 minutes. Ce résultat a été obtenu dans trois expériences conséculives.

ll a paru possible d'oblenir des conditions encore meilleures en modifiant le dispositif de manière à faire porter l'absorption sur des écrans qui ne subissent pas le refroidissement. Pour cela on a remis à nouveau l'ampoule dans le tube d'aluminium de $0, \overline{\mathrm{mm}} \mathrm{d}$ 'épaisseur, mais, en revanche, le tube central de la chambre a été remplacé par un tube de laiton de $2 \mathrm{~mm}$ d'épaisseur. La diminution est alors devenue inférieure à 1 pour 1000. Ce dispositif a été adopté pour les expériences avec l'hỷdrogène liquide.

Expériences définitives. - Les expériences o:at été exécutécs à Lejde, du 20 au 2 Ju juillet 1911.

Le courant d'ionisation dans la chambre principale élait mesuré pac 1100 unités arbitraires (énviron 10 unités E. S.). L'intensité du courant de compensation était régléc de manière à être un peu plus grande, la différence alteignant au plus 20 unités, soit environ 2 pour 100. Les rajons utilisés étaicut le's rayons $\gamma$.

Deux expériences avec l'hỵdrogène liquide ont pu être effectuées.

Dans ces expériences, ainsi que nous l'avons exposé plus haut, la chambre froide était remplie de gaz hydrogène sec, et les cond nsialions autour de l'ampoule étriient évitíes

Lans la première expérience, le courant mesuré, égal d'abord à 10,9 unités, a pris la valeur de 14,7 unités après l'introduction de l'hydrogène liquide, laquelle a demandé 15 minutes. Cette variation correspond à une diminution du courant principal, égale à 5,4 pour 1000 .

Dans la deuxième expérience, le courant mesuré était de $18, \overline{\bar{s}}$ unilés, et une bonne stabilité élait 
obtenue, les variations des mesures pendant une demi-heure n'alteignant pas 0.1 pour 1000 du courant principal. L’hyddrogène liquide ayant alors été introduit, des mesures très concordantes faites pen. dant une demi-heure ont donné pour la valeur du courant 18,כ unités, et après une heure, 18,2 unités. On peut donc affirmer que dans cette expérience, qui semble faite dans de très bonnes conditions, le refroidissement n'a pas donné lieu à une variation du courant principal qui puisse être évaluéc à 0,2 pour 1000 .

Une expérience a aussi été faite à la température de l'oxygène liquide. Le courant mesuré était de 1,8 unité. Les mesures faites pendant une heure à la température de l'oxygène liquide ont donmé pour la valeur du courant mesuré 2,6 unités, ce qui correspond à une diminution de 0,7 pour 1000 sur le courant principal.

Il aurait été désirable de faire un plus grand nombre d'expériences et aussi de les poursuivre pendant un temps plus long. Néanmoins, en considérant suriout celle des expérienecs qui parail la meilleure, il semble légitime d'aftirmer que le refroidlissement du radium à la température de l'hylrogène liquide $\left(20^{\circ}, 3\right.$ absolus) pendant un temps qui ne dépasse pas une heure et demie, ne donne pas lieu à une variation de ragonnement $\gamma$ qui puisse atteindre 1 pour 1000 , et probablement même 0,2 pour 1000 .

Il est donc probable d'aprìs cela qu'il n'existe pas, à la précision indiquéc, d'effet instantané ou très rapide de cet abaissıment de température sur l'émanation ou le dépôt actif à évolution rapide (Radium A, $B$ et $C$ ). Mais un effet sur le radium lui-même ou un effet se produisant assez lentement sur ses dérivés, n'aurait pu être mis en évidence dans ces expériences.

Expériences avec le polonium. - Quelques essais ont été faits à Paris pour étudier l'action des basses températures sur le rayonnement du polonium. L'expérience a seulement été faite avec l'air liquide. Elle présente certaines difficultés. Une plaque portant un dépôt de polonium était placée au fond d'un long tube de verre qui pouvait être plongé dans l'air liquide. Cette plaque agissait, au travers d'un éaran d'aluminium mince fermant le tube, sur une chambre d'ionisation étanche, dans laquelle les rayons du polonium étaient absorbés par l'air. Un vide aussi bon que possible était fait dans le tube à polonium; ensuite, on améliorait encore le vide en plongeant dans l'air liquide un tube latéral, adapté au premier, et contenant une petite quantité de charbon. La mesure du rajonnement était faite à la température ordinaire, et ensuite elle était répétée pendant que le fond du tube plongreait dans l'air liquide. Dans ces expériences, on a observé, lors du refroidissement, des diminutions de courant de grandeur variable.

Ces variations se sont montrées d'autant moins importantes que les précautions pour obtenir et maintenir un vide parfait étaient plus grandes. Il est donc très probable qu'elles étaient dues uniquement à la condensation sur le polonium des traces de matières gazeuses présentes dans l'appareil.

Des expériences faites à Leyde arce un appareil provisoire plongé dans l'hydrogène liquide nous ont conduits à la conclusion qu'on pourrait arriver à éliminer tout à fait ces condensations même dans l'hydrogène liquide en faisant usage d'une chambre d'ionisation remplie d'hydrogène, et d'un tube latéral à charbon plongé dans l'hydrogène liquide.

Conclusions. - Il résulte de l'ensemble de ces expériences qui ne sont malheureusement pas aussi complètes que nous l'aurions désiré, que l'indépendance du rayonnement de la température se trouve confirmée dans des limites plus étendues que précédemment. En même temps, les expérienres ont mis en évidence les causes d'erreur avec lesquelles il faut compter quand on veut pousser loin la précision des mesures à très basse température.

[. Hanuscrit reçu le 8 juin 1915. ] 